\title{
Heavy quarkonia properties from a hard-wall confinement potential model with conformal symmetry perturbing effects
}

\author{
Ahmed Al-Jamel \\ Physics Department, Al Al-Bayt University, Mafraq 25113, Jordan \\ Email: aaljamel@gmail.com or aaljamel@aabu.edu.jo
}

\begin{abstract}
Heavy $c \bar{c}$ and $b \bar{b}$ quarkonia are considered as systems confined within a hard-wall potential shaped after a linear combination of a cotangent- with a square co-secant function. Wave functions and energy spectra are then obtained in closed forms in solving by the Nikiforov-Uvarov method the associated radial Schrödinger equation in the presence of a centrifugal term. The interest in this potential is that in one parametrization it can account for a conformal symmetry of the strong interaction, and in another for its perturbation, a reason for which we here employ it to study status of conformal symmetry in the heavy flavor sector. The resulting predictions on heavy quarkonia mass spectra and root mean square radii are compared with the available experimental data, as well as with predictions by other theoretical approaches. We observe that a relatively small conformal symmetry perturbing term in the potential suffices to achieve good agreement with data.
\end{abstract}

PACS: 12.39.Pn (potential models) 03.65.Ge (Solutions of wave equation:bound states) 02.30.Ik (Integrable systems) 14.40.Lb (Charmed mesons) 14.40.Nd (Bottom mesons)

\section{Introduction}

Following the remarkable recent discovery of the Higgs boson at the Large Hadron Collider (LHC), further open questions rapidly moved to the focus of the experimental projects of this leading experimental facility, among them the study of the production mechanisms of heavy quarkonia (bound states of heavy quark and heavy antiquark), a process whose exploration is expected to provide significant new insights into our understanding of the strong interaction dynamics (for details one can refer to [1],[2],[3],[4] and references therein). Because of their heavy masses $\left(m_{q} \gg \Lambda_{Q C D}\right)$, the dynamical description of these systems grows more complex. The field theoretical perturbative methods may not always be efficient in that regard, a circumstance that can be remedied to some extent by the employment of effective degrees of freedom such as constituent quarks, interacting via nonrelativistic potentials. In the last two decades, several quark potential models have been worked out for the sake of data evaluation on heavy quarkonia, which are based on interactions such as the Cornell and Martin potentials, the Cornell plus quadratic potential, among others. The potentials used in the aforementioned models reflect to some extent the two basic features of strong interaction, asymptotic freedom, and confinement, predominantly soft walled. However, none of them relates to the conformal $S O(2,4)$ symmetry of strong interaction. The latter is best established at the kinematic level in the regime of the asymptotic freedom where the running coupling becomes practically vanishing, causing that the constituent quarks start gradually loosing their gluon "dressings", evolving to partons, the free matter fields in the QCD Lagrangians. The parton masses in the unflavored sector are sufficiently small and allow conformal symmetry to acquire importance there. Moreover, recently experimental hints have been obtained also on possible viability of a dynamical conformal symmetry in the infrared regime through the observed walking of the strong coupling towards a fixed value at origin [5]. For the sake of description of this phenomenon a quark potential is required that captures the features of the conformal symmetry, in addition to confinement and asymptotic freedom. A properly parameterized linear combination of a cotangent- and a squared co-secant functions, known under the name of the "trigonometric Rosen-Morse potential" [6], also managed by the super-symmetric quantum mechanics, has been reported in [7] to serve this purpose. In the latter work, the unflavored mesons have been studied and a fairly well agreements between predictions and data on the spectra could be found. For the heavy flavors, one expects the conformal symmetry to be violated by the large masses of the charmed and bottom quarks, and it is an intriguing task to identify a potential parameter as a signature for 
such a violation. The interaction under discussion is in addition a hard walled potential in so far as it is of an infinite depth, and the associated wave functions are vanishing in the outer region. The goal of the present work is twofold. On the one side, we wish to explore the effect of the hard walls on the properties of the heavy $(c \bar{c})$ and $(b \bar{b})$ quarkonia, and on the other, to shad some light on the status of conformal symmetry in this sector. In order to account for the second effect, we here extend the trigonometric Rosen-Morse potential applied in [7] to the description of a good dynamical conformal symmetry by a properly designed term that triggers the perturbation of the latter.

Before proceeding further, we like to notice that in being hard walled, the potential under discussion, has frequently found applications to the studies of systems featuring confinement and ranging from quantum dots [8], over Coulomb fluids [9], to hadron structure [7],[10]. Furthermore, in [12], the same potential has been considered as belonging to a broader class of trigonometric interactions, to which exact and approximate solutions have been found by means of the asymptotic iteration method. In addition, in [13], the trigonometric Rosen-Morse potential and its exact solutions have served as a point of departure towards the constructions of new potentials by subjecting it to transformations for which bound states spectra and the corresponding wave functions have been calculated. Finally, in [14] the $q$ deformed Rosen-Morse potential (in its hyperbolic version) has been defined and used as an improved model to the Tietz potential in investigations of diatomic molecules.

We here employ a version of the trigonometric Rosen-Morse potential within the framework of non-relativistic quantum mechanics to generate heavy quarkonia spectra and wave functions in three dimensional space.

The article is structured as follows. In the next section we solve the potential problem of interest by the aid of the Nikiforov-Uvarov method. Comparison with available experimental data and related theoretical calculation are presented in section 3 . The text closes with a brief summary.

\section{Heavy quarkonia potential problem and solutions}

One of the most popular techniques for solving elliptic differential equations, as are the stationary Schrödinger equations, has been developed by Nikiforov and Uvarov [15], and will be abbreviated in what follows by NU. Its most essential technical aspects are briefly highlighted in the subsequent section, for the sake of self sufficiency of the presentation.

\subsection{The Nikiforov-Uvarov method}

The method is based on the assumption that the stationary one-dimensional Schrödinger equation can be cast in the form of the generalized hyper-geometric equation as,

$$
\psi^{\prime \prime}(s)+\frac{\tilde{\tau}(s)}{\sigma(s)} \psi^{\prime}(s)+\frac{\tilde{\sigma}(s)}{\sigma^{2}(s)} \psi(s)=0,
$$

by the aid of some suitable point-canonical transformation. Here, $\tilde{\tau}(s)$ is a polynomial of at most first degree, while $\sigma(s)$ and $\tilde{\sigma}(s)$ are polynomials of at most second degree [15]. Utilizing factorization,

$$
\psi(s)=\phi(s) y(s)
$$

Eq.(1) allows for a transformation to an equation of the standard hyper-geometric type:

$$
\sigma(s) y^{\prime \prime}+\tau(s) y^{\prime}+\lambda y=0
$$

where $\tau(s)$ has been defined as

$$
\tau(s)=\tilde{\tau}(s)+2 \pi(s)
$$

while the $\pi(s)$ function is given by,

$$
\pi(s)=\frac{\sigma^{\prime}(s)-\tilde{\tau}(s)}{2} \pm \sqrt{\left(\frac{\sigma^{\prime}(s)-\tilde{\tau}(s)}{2}\right)^{2}-\tilde{\sigma}(s)+k \sigma(s)}
$$


The latter is supposed to be a polynomial of first degree at most, so that the expression under the square root in Eq.(5) could take the shape of a square of a polynomial of first degree. The parameters $\lambda$ and $k$ then satisfy the condition,

$$
\lambda=k+\pi^{\prime}(s)
$$

Provided the sign of the derivative of the $\tau(s)$ function is negative, the equation (3) can be shown to have polynomial solutions $y_{n}(s)$, generated by the Rodrigues formula according to,

$$
y_{n}(s)=\frac{B_{n}}{\rho(s)} \frac{d^{n}}{d s^{n}}\left[\sigma^{n}(s) \rho(s)\right]
$$

Here, $B_{n}$ is a normalization constant, and $\rho(s)$, termed to as density-, or weight function, must satisfy the condition

$$
(\sigma(s) \rho(s))^{\prime}=\tau(s) \rho(s)
$$

Moreover, for this case one finds an $n$ dependent $\lambda$ according to,

$$
\lambda_{n}=-n \tau^{\prime}(s)-\frac{n(n-1)}{2} \sigma^{\prime \prime}(s), \quad n=0,1,2, \ldots,
$$

With that, the function $\phi(s)$ is found to satisfy

$$
\frac{\phi(s)^{\prime}}{\phi(s)}=\frac{\pi(s)}{\sigma(s)}
$$

Equating Eq.6 with Eq.96, it can be shown to allow to obtain the energy eigenvalues of the Schrödinger equation.

\subsection{Solving the Schrödinger equation with the trigonometric Rosen-Morse potential by the aid of the NU method}

We are interested in investigating quantum systems confined in the trigonometric Rosen-Morse potential, to be abbreviated by "tRM", of the form given by [7],[10] (here in dimensionless units)

$$
V\left(\frac{r}{a}\right)=-V_{0} \cot \left(\frac{r}{a}\right)+d(d+1) \csc ^{2}\left(\frac{r}{a}\right)+a^{2} \frac{\ell(\ell+1)}{r^{2}}, \quad 0<r<a \pi,
$$

with $\ell$ standing for the angular momentum value. In the approximation,

$$
V\left(\frac{r}{a}\right) \approx-V_{0} \frac{a}{r}+V_{0} \frac{r}{3 a}+[\ell(\ell+1)+d(d+1)] \frac{a^{2}}{r^{2}},
$$

it reproduces the functional form of a potential of a frequent use [17] in the spectroscopy of quarkonia. This approximation emerges from keeping the lowest terms in the series expansions of,

$$
-\cot \left(\frac{r}{a}\right) \approx-\frac{a}{r}+\frac{1}{3} \frac{r}{a}
$$

and

$$
\csc ^{2}\left(\frac{r}{a}\right) \approx \frac{1}{\frac{r^{2}}{a^{2}}}-\frac{1}{15} \frac{r^{2}}{a^{2}},
$$

respectively. Within this context, the potential in (11) can be viewed as an upgrade of the one in (12). The stationary Schrödinger equation we are interested in here reads

$$
\begin{aligned}
\mathcal{H}_{t R M}\left(\frac{r}{a}\right) u_{n \ell}\left(\frac{r}{a}\right) & =E u_{n \ell}\left(\frac{r}{a}\right), \\
\mathcal{H}_{t R M}\left(\frac{r}{a}\right) & =-\left[\frac{\hbar^{2}}{2 \mu} \frac{d^{2}}{d r^{2}}+\frac{\hbar^{2}}{2 \mu a^{2}}\left[V_{0} \cot \left(\frac{r}{a}\right)-d(d+1) \csc ^{2}\left(\frac{r}{a}\right)-\frac{\ell(\ell+1)}{2 \mu r^{2}}\right]\right] .
\end{aligned}
$$


With the aim to arrive at exact solutions, we shall approximate the centrifugal term in the latter equation as,

$$
\frac{1}{r^{2}} \approx \frac{1}{a^{2} \sin ^{2}\left(\frac{r}{a}\right)} \equiv \frac{1}{a^{2}} \csc ^{2}\left(\frac{r}{a}\right), \quad \frac{r}{a}<<1 .
$$

In effect, the wave equation to be solved takes in dimensionless units the shape,

$$
\left[a^{2} \frac{d^{2}}{d r^{2}}+\frac{2 \mu c^{2} a^{2}}{\hbar^{2} c^{2}} E+V_{0} \cot \left(\frac{r}{a}\right)-d(d+1) \csc ^{2}\left(\frac{r}{a}\right)-\frac{\ell(\ell+1)}{\sin ^{2}\left(\frac{r}{a}\right)}\right] u_{n \ell}\left(\frac{r}{a}\right)=0 .
$$

Within the above context, the equation (17) can be interpreted in a twofold way.

- Without the approximation in (16) it represents the radial part of the flat-space Suchödinger equation with the trigonometric Rosen-Morse potential in the presence of a centrifugal term.

- With this approximation, same equation can be read as equation in the angular variable, $\chi=r / a$, where $a$ plays the role of a spherical radius, while $r$ acquires meaning of the arc on a great circle, read off from the "North pole". The fact is that the term $\ell(\ell+1) \csc ^{2} \chi$ represents the centrifugal barrier on the three dimensional sphere, $S^{3}$, whose isometry $S O(4)$, is the maximal compact subgroup of the conformal group, $S O(2,4)$, a reason for which the equation for $d=0$ describes dynamical conformal symmetry. The additional $d(d+1) \csc ^{2}(r / a)$ term could be interpreted within this context as a conformal symmetry perturbation that alters the shape of the wave functions (see discussion to the end of section 3 below).

Now, with the help of $\csc ^{2}\left(\frac{r}{a}\right)=1+\cot ^{2}\left(\frac{r}{a}\right)$ and the substitutions

$$
\epsilon=\frac{2 \mu}{\hbar^{2}} E, \quad V_{c}=\frac{\hbar^{2} V_{0}}{2 \mu a^{2}}, \quad U_{0}=\frac{V_{0}}{a^{2}}, \quad \gamma=\frac{1}{a^{2}}(d(d+1)+\ell(\ell+1))
$$

where $V_{c}$ is the amplitude of the cotangent part of the potential in units of $\mathrm{MeV}$, while the rest of variables are in units of $\mathrm{fm}^{-2}$, Eq. (17) becomes

$$
\left[\frac{d^{2}}{d r^{2}}+\left(\epsilon+U_{0} \cot \left(\frac{r}{a}\right)-\gamma-\gamma \cot ^{2}\left(\frac{r}{a}\right)\right)\right] u_{n \ell}\left(\frac{r}{a}\right)=0,
$$

Introducing a suitable transformation as $u_{n \ell}(r / a)=\sin (r / a) f(r / a)$, which ensures that $u_{n \ell}(0)=u_{n \ell}(\pi)=0$, followed by a change of variable, $y=\cot \left(\frac{r}{a}\right)$, and upon making use of the Ansatz [12]

$$
f(y)=\left(1+y^{2}\right)^{\frac{\alpha}{2}} e^{-\beta \operatorname{arccot}(y)} g(y), \quad-\infty<y<\infty,
$$

the equation (19) is found to take the following shape,

$\frac{d^{2} g(y)}{d y^{2}}+\frac{2(\alpha y+\beta)}{1+y^{2}} \frac{d g(y)}{d y}+\frac{\left(\alpha(\alpha-1)-a^{2} \gamma\right) y^{2}+\left(2 \beta(\alpha-1)+U_{0} a^{2}\right) y+\alpha+a^{2} \epsilon+\beta^{2}-a^{2} \gamma-1}{\left(1+y^{2}\right)^{2}} g(y)=0$.

The freedom in the selection of $\alpha$ and $\beta$ in Eq. (21) allows us to choose:

$$
\begin{aligned}
2 \beta(\alpha-1)+U_{0} a^{2} & =0 . \\
a^{2} \epsilon-(\alpha-1)^{2}+\beta^{2} & =0,
\end{aligned}
$$

then Eq. (21) becomes

$$
\frac{d^{2} g(y)}{d y^{2}}+\frac{2(\alpha y+\beta)}{1+y^{2}} \frac{d g(y)}{d y}+\frac{\left(\alpha(\alpha-1)-a^{2} \gamma\right)\left(1+y^{2}\right)}{\left(1+y^{2}\right)^{2}} g(y)=0 .
$$


In making now use of the equations (1)-(6) from the NU method, allows for the following identifications: $\tilde{\tau}(y)=$ $2(\alpha y+\beta), \sigma(y)=1+y^{2}, \tilde{\sigma}(y)=\left(\alpha(\alpha-1)-a^{2} \gamma\right)\left(1+y^{2}\right)$. Therefore, we can proceed executing the further prescriptions of the NU method. In so doing, we first find the function $\pi(y)$ in (5) expressed as,

$$
\pi(y)=(1-\alpha) y-\beta \pm \sqrt{k\left(y^{2}+1\right)-\left(\alpha(\alpha-1)-a^{2} \gamma\right)\left(y^{2}+1\right)+((1-\alpha) y-\beta)^{2}} .
$$

The k-roots are $k_{1}=-\alpha+\alpha^{2}-a^{2} \gamma$ and,$k_{2}=\alpha-\beta^{2}-\gamma-1$. Therefore we have four possible choices for $\pi(y)$. The only choice that gives physically acceptable solutions is the one with $k_{1}$ corresponding to negative sign in Eq. (4). The results is $\pi(y)=0$. The associated function $\tau$ is then $\tau(y)=2 \alpha y+2 \beta$. This function has a negative sign derivative only if $\alpha<0$. In this case we obtain:

$$
\alpha^{2}+(2 n-1) \alpha+n(n-1)-a^{2} \gamma=0 .
$$

Solving the latter equation we obtain

$$
\alpha=-n+\frac{1}{2} \pm \frac{1}{2} \sqrt{4 a^{2} \gamma+1}
$$

The constraint $\alpha<0$ amounts to the following choice for $\alpha$ :

$$
\alpha=-n+\frac{1}{2}-\frac{1}{2} \sqrt{4 a^{2} \gamma+1}
$$

which is always negative for any $n \geq 0$ and $\gamma \geq 0$. Substituting for $\gamma$ from Eq. (18), we obtain

$$
\alpha=-n+\frac{1}{2}-\frac{1}{2} \sqrt{4 d(d+1)+4 \ell(\ell+1)+1} .
$$

Plugging this in Eq. (22), we obtain the energy eigenvalues as

$$
E_{n \ell}=\frac{\hbar^{2}}{2 \mu a^{2}}(-\alpha-1)^{2}-\frac{\hbar^{2} V_{0}^{2}}{8 \mu a^{2}(-\alpha-1)^{2}} .
$$

Here $n, \ell=0,1,2, \ldots$. Note that this energy formula admits as well positive as negative value of $V_{0}$ since this parameter enters the energy formula through its square, $V_{0}^{2}$. The special case of $d=0$ amounts to,

$$
E_{n \ell}=\frac{\hbar^{2}}{2 \mu a^{2}}(n+\ell+1)^{2}-\frac{\hbar^{2} V_{0}^{2}}{8 \mu a^{2}(n+\ell+1)^{2}},
$$

and reproduces the related result in [12, 10]. For small $d$, the expansion of Eq. (29) around $d=0$ yields

$$
\begin{aligned}
E_{n \ell} & \approx \frac{\hbar^{2}}{2 \mu a^{2}}(n+\ell+1)^{2}-\frac{\hbar^{2} V_{0}^{2}}{8 \mu a^{2}(n+\ell+1)^{2}} \\
& +d\left(\frac{2 \hbar^{2}\left(\sqrt{\ell^{2}+\ell+1}+n+\frac{1}{2}\right)}{a^{2} \mu \sqrt{\ell^{2}+\ell+1}}+\frac{2 \hbar^{2} V_{0}^{2}}{a^{2} \mu \sqrt{\ell^{2}+\ell+1}\left(\sqrt{\ell^{2}+\ell+1}+2 n\right)^{3}}\right),
\end{aligned}
$$

which will be used later.

For the corresponding eigenfunctions, one can easily verify that, using Eq. [19, the following equations hold valid,

$$
\begin{aligned}
& \phi(y)=\text { constant } \\
& \rho(y)=\left(1+y^{2}\right)^{\alpha-1} e^{2 \beta \arctan (y)} \\
& Y_{n}(y)=B_{n} e^{-2 \beta \arctan (y)}\left(1+y^{2}\right)^{1-\alpha} \frac{d^{n}}{d y^{n}}\left(\left(1+y^{2}\right)^{n+\alpha-1} e^{2 \beta \arctan (y)}\right),
\end{aligned}
$$


hold valid. From that, the Rodrigues formula that generates the polynomials $g_{n}(y)$ is concluded as

$$
g_{n}(y)=B_{n}\left(1+y^{2}\right)^{1-\alpha} e^{-2 \beta \arctan (y)} \frac{d^{n}}{d y^{n}}\left(\left(1+y^{2}\right)^{n+\alpha-1} e^{2 \beta \arctan (y)}\right)
$$

where $B_{n}$ are the normalization constants. Note that it depends on the $\arctan (y)$. According to this formula, and ignoring the normalization constants, we have

$$
\begin{aligned}
g_{0}(y) & =1 \\
g_{1}(y) & =2(\alpha y+\beta) \\
g_{2}(y) & =2(\alpha+1)(2 \alpha+1) y^{2}+4(2 \alpha \beta+\beta) y+2\left(\alpha+2 \beta^{2}+1\right) \\
g_{3}(y) & =4(\alpha+1)(\alpha+2)(2 \alpha+3) y^{3}+12(\alpha+1)(2 \alpha+3) \beta y^{2}+4\left(6(\alpha+1) \beta^{2}\right. \\
& +3(\alpha+1)(\alpha+2)) y+4\left(3 \alpha \beta+2 \beta^{3}+5 \beta\right) \\
\ldots & =\ldots
\end{aligned}
$$

which coincide with the related expressions reported in [12]. These polynomials $g_{n}(y)$ are related to the known as Romanovski polynomials $R_{n}^{\beta, \alpha}(y)$, which are defined by the Rodrigues formula [16]

$$
R_{n}^{\beta, \alpha}(y)=\frac{1}{\omega^{\beta, \alpha}(y)} \frac{d^{n}}{d y^{n}}\left(\omega^{\beta, \alpha}(y) S(y)^{n}\right)
$$

where

$$
\omega^{\beta, \alpha}(y)=\left(1+y^{2}\right)^{\alpha-1} \mathrm{e}^{-\beta \operatorname{arccot}(y)}
$$

is the weight function, equivalent to $\rho(y)$ in the NU method, and $S(y)=1+y^{2}$. This can be seen if we use

$$
\arctan (y)+\operatorname{arccot}(y)=\frac{\pi}{2}, \quad \operatorname{Re}[y] \geq 0
$$

and noting that

$$
\begin{aligned}
\omega^{2 \beta, \alpha}(y) & =\left(1+y^{2}\right)^{\alpha-1} \mathrm{e}^{-2 \beta \operatorname{arccot}(y)} \\
& =\left(1+y^{2}\right)^{\alpha-1} \mathrm{e}^{2 \beta \arctan (y)-\beta \pi}
\end{aligned}
$$

and thus

$$
\begin{aligned}
R_{n}^{2 \beta, \alpha}(y) & =\left(1+y^{2}\right)^{1-\alpha} \mathrm{e}^{-2 \beta \arctan (y)-\beta \pi} \frac{d^{n}}{d y^{n}}\left(\left(1+y^{2}\right)^{n+\alpha-1} \mathrm{e}^{2 \beta \arctan (y)+\beta \pi}\right) \\
& =\left(1+y^{2}\right)^{1-\alpha} \mathrm{e}^{-2 \beta \arctan (y)} \frac{d^{n}}{d y^{n}}\left(\left(1+y^{2}\right)^{n+\alpha-1} \mathrm{e}^{2 \beta \arctan (y)}\right) \\
& =g_{n}(y) \text { (up to a multiplicative constant) }
\end{aligned}
$$

The orthonormalization of Romanovski polynomials is:

$$
\int_{-\infty}^{\infty} R_{n}^{\beta, \alpha}(y) R_{m}^{\beta, \alpha}(y) \omega^{\beta, \alpha}(y) d y=B_{n}^{2} \delta_{n m}, \quad \text { iff } \quad m+n<1-2 \alpha
$$

which leads to the orthonormalization condition

$$
\int_{-\infty}^{\infty} g_{n}(y) g_{m}(y)\left(1+y^{2}\right)^{\alpha-1} e^{2 \beta \arctan (y)} d y=B_{n}^{2} \delta_{n m}, \quad \text { iff } \quad m+n<1-2 \alpha .
$$


Thus, for the case when the polynomial's parameters do not depend on its degree, only a finite number of polynomials, namely those satisfying the constraint $m+n<1-2 \alpha$, are orthogonal, a circumstance known in literature under the name of " finite orthogonality". However, for the case considered here, in which the polynomial parameters depend on the polynomial degrees in accord with the equations (28) and $\beta$ from (22), an infinite orthogonality is encountered [16]. The reduced wave function can now be written in a closed form as a function of $r / a$ as

$$
\begin{aligned}
u_{n \ell}\left(\frac{r}{a}\right) & =B_{n} \sin \left(\frac{r}{a}\right) f\left(\frac{r}{a}\right) \\
& =B_{n} \sin \left(\frac{r}{a}\right)\left(1+\cot ^{2}\left(\frac{r}{a}\right)\right)^{\frac{\alpha}{2}} \mathrm{e}^{-\beta \operatorname{arccot}\left(\cot \left(\frac{r}{a}\right)\right)} g_{n}\left(\cot \left(\frac{r}{a}\right)\right) \\
& =B_{n} \sin \left(\frac{r}{a}\right)\left(1+\cot ^{2}\left(\frac{r}{a}\right)\right)^{1-\frac{\alpha}{2}} \mathrm{e}^{-2 \beta \arctan \left(\cot \left(\frac{r}{a}\right)\right)-\beta \frac{r}{a}} \\
& \times \frac{d^{n}}{d\left(\cot \left(\frac{r}{a}\right)\right)^{n}}\left(\left(1+\cot \left(\frac{r}{a}\right)^{2}\right)^{n+\alpha-1} \mathrm{e}^{\left.2 \beta \arctan \left(\cot \left(\frac{r}{a}\right)\right)\right)} .\right.
\end{aligned}
$$

where we have used Eq. (42) and the fact $\cot ^{-1}\left(\cot \left(\frac{r}{a}\right)\right)=\frac{r}{a}$. Here the value of $\beta$ is

$$
\beta=\frac{-V_{0}}{2(\alpha-1)}
$$

which must be positive to ensure the exponential fall-off of the wave functions needed for convergence of various integrals related to physical observable. This condition is already satisfied as $\alpha$ is found to be negative for the applicability of the method.

\section{Analyzing data on heavy quarkonia}

\subsection{Mass spectra}

The mass spectra of heavy quarkonia can be produced using the formula:

$$
M_{q \bar{q}}=m_{q}+m_{\bar{q}}+E_{n \ell},
$$

where $E_{n \ell}$ is given by Eq. (29), $m_{q}$ and $m_{\bar{q}}$ are the masses of the constituent quarks, and anti-quarks, respectively. The reduced mass $\mu$ that appears in Eq. (29) is defined in the standard way as $\mu=\frac{m_{q} m_{\bar{q}}}{m_{q}+m_{\bar{q}}}$. For $b \bar{b}$ and $c \bar{c}$ systems, we adopt the numerical values of these masses as $m_{b}=4.67 \mathrm{GeV}$ for bottomonium, and $m_{c}=1.50 \mathrm{GeV}$ for charmonium. Then, the corresponding reduced masses are $\mu_{b}=2.335 \mathrm{GeV}$ and $\mu_{c}=0.75 \mathrm{GeV}$, respectively. The magnitude $V_{0}$ of the cotangent term in (11) has been parameterized as $V_{0}=\alpha_{s} N_{c}$ with $\alpha_{s}$ being the value of the strong coupling for $m_{c}$, and $N_{c}=3$ the number of colors. The values of $\alpha_{s}$ at the heavy quark masses of interest have been measured with varying precision and reported for example in [17], [21], [22], [23], [24]. In the present study we opted in favor of using in the numerical calculations a common reasonable averaged value of $\alpha_{s}=0.2$. The potential parameters $a$ and $d$ can then be obtained by simultaneous fitting available experimental data on excited heavy flavor mesons. By adjusting masses of $1 S$ and $2 P$ states of $b \bar{b}$ and $c \bar{c}$ mesons, we extracted the values of these parameters for each one of the two sectors. Then, the remaining states in each one of the two spectra are predicted using the obtained parameters according to Eqs. (47) and (29). The results are presented in Tables (1,2). These show that our energy formula can reproduce the masses in pretty good agreement with data. In Figures 2, we plotted the trigonometric Rosen-Morse potential with the adjusted parameters for the cases of an $S$-wave-, and a $P$ wave state for charmonium. It is visible how the corresponding potentials bend strongly upwards from both sides of the interval, a behavior that reflects the strong confinement of the systems under study. The bending of the trigonometric Rosen-Morse potential for zero angular momenta is due to the $d(d+1) \csc ^{2}(r / a)$ term and therefore to the conformal symmetry perturbation. 
Table 1: Mass spectra for $c \bar{c}$ in GeV.According to [7] the magnitude $V_{0}$ of the cotangent term in (11) has been parameterized as $V_{0}=\alpha_{s} N_{c}$ with $\alpha_{s}$ being the value of the strong coupling for $m_{c}$, and $N_{c}=3$ the number of colors. Using $\alpha_{s}=0.2$, the dimensional magnitude emerges as $V_{c}=0.050 \mathrm{GeV}$ for the reduced mass of $\mu_{c}=0.75 \mathrm{GeV}$ and $m_{c}=1.5 \mathrm{GeV}$. Furthermore, $d=0.109, a=2.822 \mathrm{GeV}^{-1}=0.56 \mathrm{fm}$. Experimental data are taken from [18, 19].

\begin{tabular}{ccccc}
\hline$n L$ & Present work & Present work $d=0$ & Experimental & Ref. [17] \\
\hline $1 \mathrm{~S}$ & 3.097 & 3.076 & 3.097 & 3.096 \\
$2 \mathrm{~S}$ & 3.371 & 3.333 & 3.686 & 3.686 \\
$1 \mathrm{P}$ & 3.347 & 3.333 & 3.525 & 3.214 \\
$3 \mathrm{~S}$ & 3.809 & 3.752 & 4.040 & 4.275 \\
$2 \mathrm{P}$ & 3.773 & 3.752 & 3.773 & 3.773 \\
$1 \mathrm{D}$ & 3.765 & 3.752 & 3.770 & 3.412 \\
$4 \mathrm{~S}$ & 4.414 & 4.339 & 4.263 & 4.865 \\
3P & 4.366 & 4.339 & - & - \\
2D & 4.355 & 4.339 & - & - \\
$1 \mathrm{~F}$ & 4.350 & 4.339 & - & - \\
\hline
\end{tabular}

Table 2: Mass spectra for $b \bar{b}$ (in GeV). According to [7] the magnitude $V_{0}$ of the cotangent term in (11) has been parameterized as $V_{0}=\alpha_{s} N_{c}$ with $\alpha_{s}$ being the value of the strong coupling for $m_{b}$, and $N_{c}=3$ the number of colors. Using $\alpha_{s}=0.2$, the dimensional magnitude emerges as $V_{c}=0.059 \mathrm{GeV}$ for the reduced mass of $\mu_{b}=2.335 \mathrm{GeV},\left(m_{b}=4.67 \mathrm{GeV}\right)$. Furthermore, $d=0.131, a=1.468 \mathrm{GeV}^{-1}=0.290 \mathrm{fm}$. Experimental data are taken from [18, 19].

\begin{tabular}{ccccc}
\hline$n L$ & Present work & Present work $d=0$ & Experimental & Ref. [17] \\
\hline $1 \mathrm{~S}$ & 9.460 & 9.430 & 9.460 & 9.460 \\
$2 \mathrm{~S}$ & 9.789 & 9.735 & 10.023 & 10.023 \\
$1 \mathrm{P}$ & 9.755 & 9.735 & 9.899 & 9.492 \\
3S & 10.312 & 10.233 & 10.355 & 10.585 \\
2P & 10.262 & 10.233 & 10.260 & 10.038 \\
1D & 10.250 & 10.233 & 10.164 & 9.551 \\
$4 \mathrm{~S}$ & 11.034 & 10.929 & 10.580 & 11.148 \\
3P & 10.967 & 10.929 & - & - \\
2D & 10.952 & 10.929 & - & - \\
$1 \mathrm{~F}$ & 10.945 & 10.929 & - & - \\
\hline
\end{tabular}



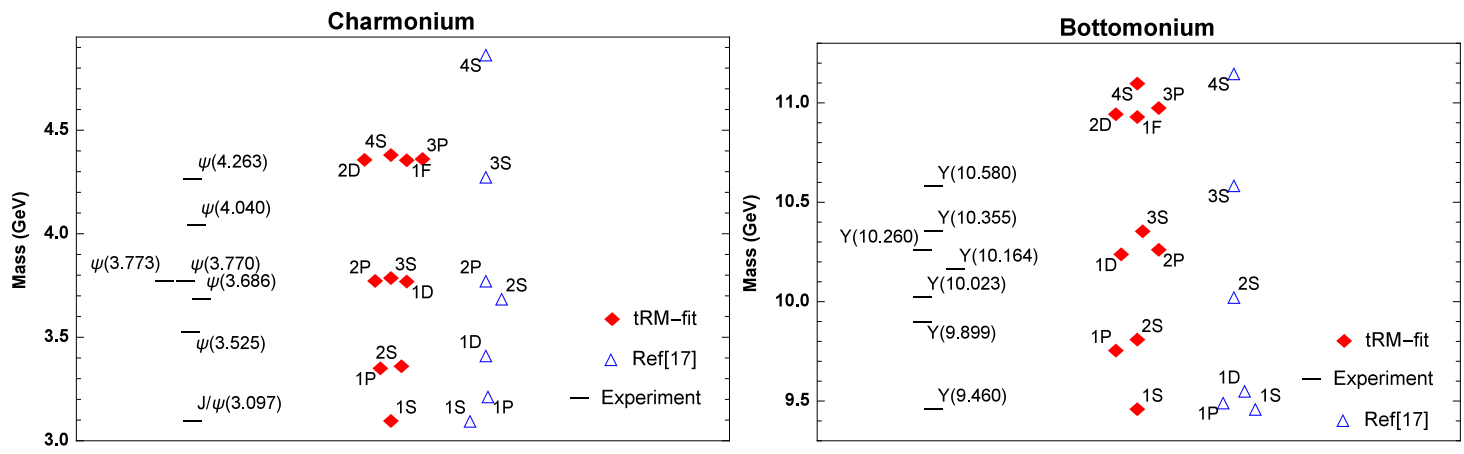

Figure 1: The predictions (red diamonds) of the charmonium (left panel) and bottomonium (right panel) spectra by the tRM potential. The mass in $\mathrm{GeV}$ is read off from the vertical axis, while the horizontal line is marginal. The experimental data are taken from [18, 19]. As a comparison we bring the theoretical predictions by [17] (blue triangles).

The fits presented in the tables show a satisfactory overall agreement between predictions and data. They also show that in each one of the cases the $d$ value is maintained surprisingly small indeed, a reason for which the predicted level splittings strongly underestimate the experimentally observed ones, although the level orderings (the signs of the splittings) come out correct. In contrast, some of the level splitting predicted by [17], like the 3S$1 D$ splitting in the $b \bar{b}$ sector, strongly overestimates the experimental data point. These observations indicate that new possibilities need to be searched for in future research with the aim of achieving a more realistic description of the level splittings. In first place we expect relevance of kinematic level splittings as they appear in a Klein-Gordon equation with the potential under discussion. Finally, for a vanishing $d$ value, a finite number of states belonging to one and the same "principal quantum number," $N=\ell+n+1$, were predicted, and perfect degeneracies among couples of the type, $2 S-1 P, 3 S-2 P-1 D$ etc with $N=2$, and $N=3$, respectively, showed up. Without entering into technical details, such occurs because the Schrödinger Hamiltonian with our potential for $d=0$, i.e. $\mathcal{H}(r / a)$ in 15 for $d=0$, is conformally symmetric due to the circumstance that it is intertwined with the Casimir invariant, $\mathcal{K}^{2}$, of the rotational group in four dimensions, $S O(4)$, the maximal compact subgroup of the conformal group $S O(2,4)$. Indeed, in this case, the parameter $a$ acquires meaning of radius of a three dimensional hyper-sphere, $S^{3}$, whose isometry is that very same $S O(4)$. Then, the $(r / a)$ variable acquires meaning of the second polar angle, here denoted by $\chi$, and used in the parametrization of $S^{3}$. The first polar angle, $\theta$, and the azimuthal angle, $\varphi$ are same as in the standard spherical harmonics. The form of this intertwinement depends on the aforementioned angles and is most simple for the case of the ground state where one can directly calculate that the following relation holds valid,

$$
\begin{aligned}
\left.e^{\frac{V_{0}}{2} \chi} \mathcal{H}(\chi)\right|_{d=0} & =\left[\mathcal{K}^{2}(\chi)+\frac{V_{0}^{2}}{4}\right] e^{\frac{V_{0}}{2} \chi}, \quad \chi:=\frac{r}{a} \\
\mathcal{K}^{2}(\chi) & =-\frac{1}{\sin ^{2} \chi} \frac{\partial}{\partial \chi} \sin ^{2} \chi \frac{\partial}{\partial \chi}+\frac{\ell(\ell+1)}{\sin ^{2} \chi}
\end{aligned}
$$

In other words, upon changing the wave function according to, $u_{n \ell}(r / a) / \sin (r / a)=\psi(r / a)$, amounts to quantum motion on the three-dimensional hyper-sphere, $S^{3}$. There, the $\ell(\ell+1) \csc ^{2}(r / a)$ term acquires meaning of the centrifugal barrier on this manifold, while the cotangent function provides a harmonic solution to the $S^{3}$ Laplacian, pretty much as the $1 / r$ function provides a harmonic solution to the regular flat space Laplacian. To the amount the isometric group of $S^{3}$ is $S O(4)$, the maximal compact group of the conformal group $S O(2,4)$, the quantum motion on $S^{3}$ perturbed by the harmonic cotangent potentials alone, necessarily obeys same symmetry as the Laplacian, namely $S O(4) \subset S O(2,4)$ in our case [7]. All these considerations follow from the principles underlying the 

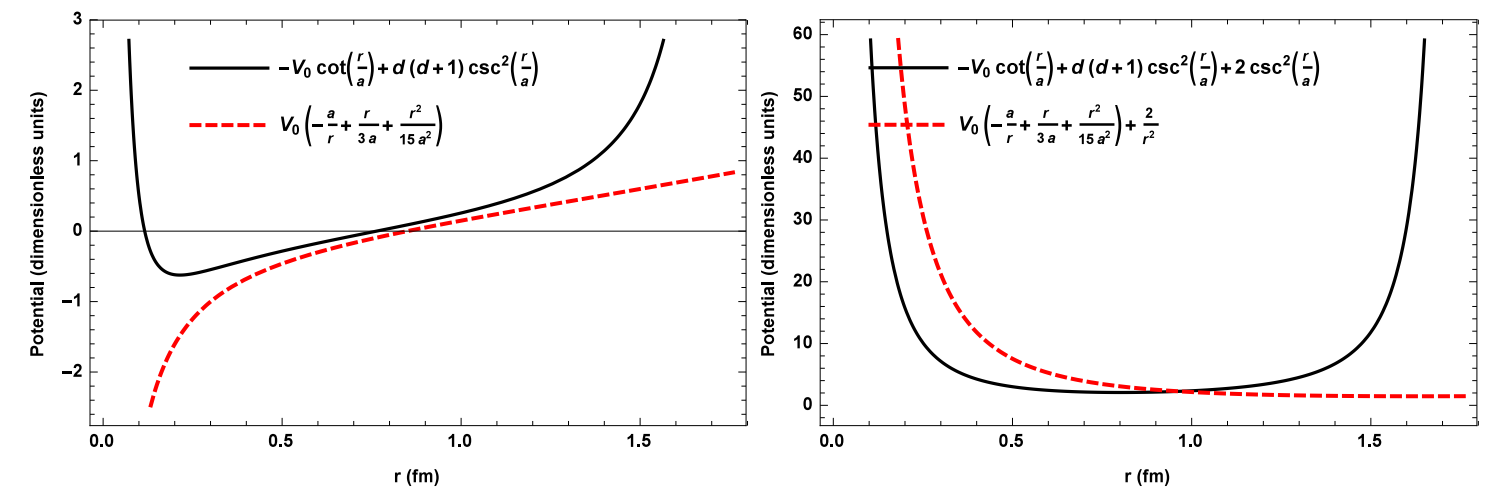

Figure 2: The hard-walled trigonometric Rosen-Morse potential (solid line) in comparison to the soft-walled Cornell+harmonic oscillator + centrifugal terms (dashed line) for the (charmonium) with parameter values of $a, d$ as given in Table (1) caption, here for $l=0$ (left panel), and $l=1$ (right panel).

mathematical discipline of potential theory on surfaces. Being con formally symmetric for $d=0$, our potential for $d \neq 0$, therefore describes a system whose conformal symmetry has been perturbatively violated through removing the degeneracies in the levels, the $S O(4)$ multiple ts, characterized by the principle quantum number $N=(n+\ell+1)$. In order to clarify the status of the symmetry, we need to compare the wave functions of the perturbed with the unperturbed case and figure out whether or not the degeneracy removal is accompanied by mixing of states from different multiplets (levels). When such mixing does not happen, then the symmetry is preserved as 'dynamical" [20].

Using the equation (45), we calculate (up to the normalization constant) the wave functions for $1 S$ and $1 P$ states for $c \bar{c}$ and $b \bar{b}$.

$$
\begin{aligned}
& u_{1 S}^{c \bar{c}}\left(\frac{r}{a}\right)=\mathrm{e}^{-\frac{0.27 r}{a}} \sin ^{1.109}\left(\frac{r}{a}\right), \\
& u_{1 P}^{c \bar{c}}\left(\frac{r}{a}\right)=\mathrm{e}^{-\frac{0.147 r}{a}} \sin ^{2.039}\left(\frac{r}{a}\right), \\
& u_{2 S}^{c \bar{c}}\left(\frac{r}{a}\right)=\mathrm{e}^{-\frac{0.142 r}{a}} \sin ^{2.109}\left(\frac{r}{a}\right)\left[0.284-2.218 \cot \left(\frac{r}{a}\right)\right] \text {, } \\
& u_{1 S}^{b \bar{b}}\left(\frac{r}{a}\right)=\mathrm{e}^{-\frac{0.265 r}{a}} \sin ^{1.131}\left(\frac{r}{a}\right) \text {, } \\
& u_{1 P}^{b \bar{b}}\left(\frac{r}{a}\right)=\mathrm{e}^{-\frac{0.146 r}{a}} \sin ^{2.048}\left(\frac{r}{a}\right), \\
& u_{2 S}^{b \bar{b}}\left(\frac{r}{a}\right)=\mathrm{e}^{-\frac{0.141 r}{a}} \sin ^{2.131}\left(\frac{r}{a}\right)\left[0.282-2.26 \cot \left(\frac{r}{a}\right)\right] .
\end{aligned}
$$

The expressions in the brackets are Romanovski polynomials of first order. The normalization constant $N_{n \ell}$ for a certain state can be calculated using the condition

$$
N_{n \ell}^{2} \int_{0}^{\pi}\left|u_{n \ell}\left(\frac{r}{a}\right)\right|^{2} 4 \pi d\left(\frac{r}{a}\right)=1,
$$

where $4 \pi$ is the integration over the solid angle. With the aim to prove the symmetry status according to the criteria discussed above, we pick up as an illustrative example the $1 S$ wave function and factorize its conformally symmetric part corresponding to $d=0$ finding,

$$
\begin{aligned}
& u_{1 S}^{c \bar{c}}\left(\frac{r}{a}\right)=\left(\mathrm{e}^{-\frac{0.30 r}{a}} \sin ^{0.109}\left(\frac{r}{a}\right)\right) \mathrm{e}^{0.03 \frac{r}{a}} \sin \left(\frac{r}{a}\right) \\
& u_{1 S}^{b \bar{b}}\left(\frac{r}{a}\right)=\left(\mathrm{e}^{-\frac{0.30 r}{a}} \sin ^{0.131}\left(\frac{r}{a}\right)\right) \mathrm{e}^{0.034 \frac{r}{a}} \sin \left(\frac{r}{a}\right) .
\end{aligned}
$$


We observe that the $d$-term in (17) solely modulates the shape of the conformally symmetric wave function, given by the expression in the round brackets, through the factor remaining outside, but does not provoke any mixing with the $2 S$ state in (51). Similar analyses can be performed with the remaining wave functions. In this manner,the conformal symmetry still maintains its viability in the heavy flavor sectors as a dynamical symmetry [20]. In Figures (3), we plotted the normalized reduced probability density $\left|u_{n \ell}(r / a)\right|^{2}$ for various states. It can be seen that the curves end in zero at the boundaries $r \in[0, a \pi]$. The smallness of the $a$ parameter is indicative of a strong spatial localization of the $q-\bar{q}$ system. As a comparison, we also plotted the spherical Bessel functions, the ground state solutions to an other hard-walled potential, the infinite spherical "square" potential. The node number $n$ is same in both cases, as it should be.
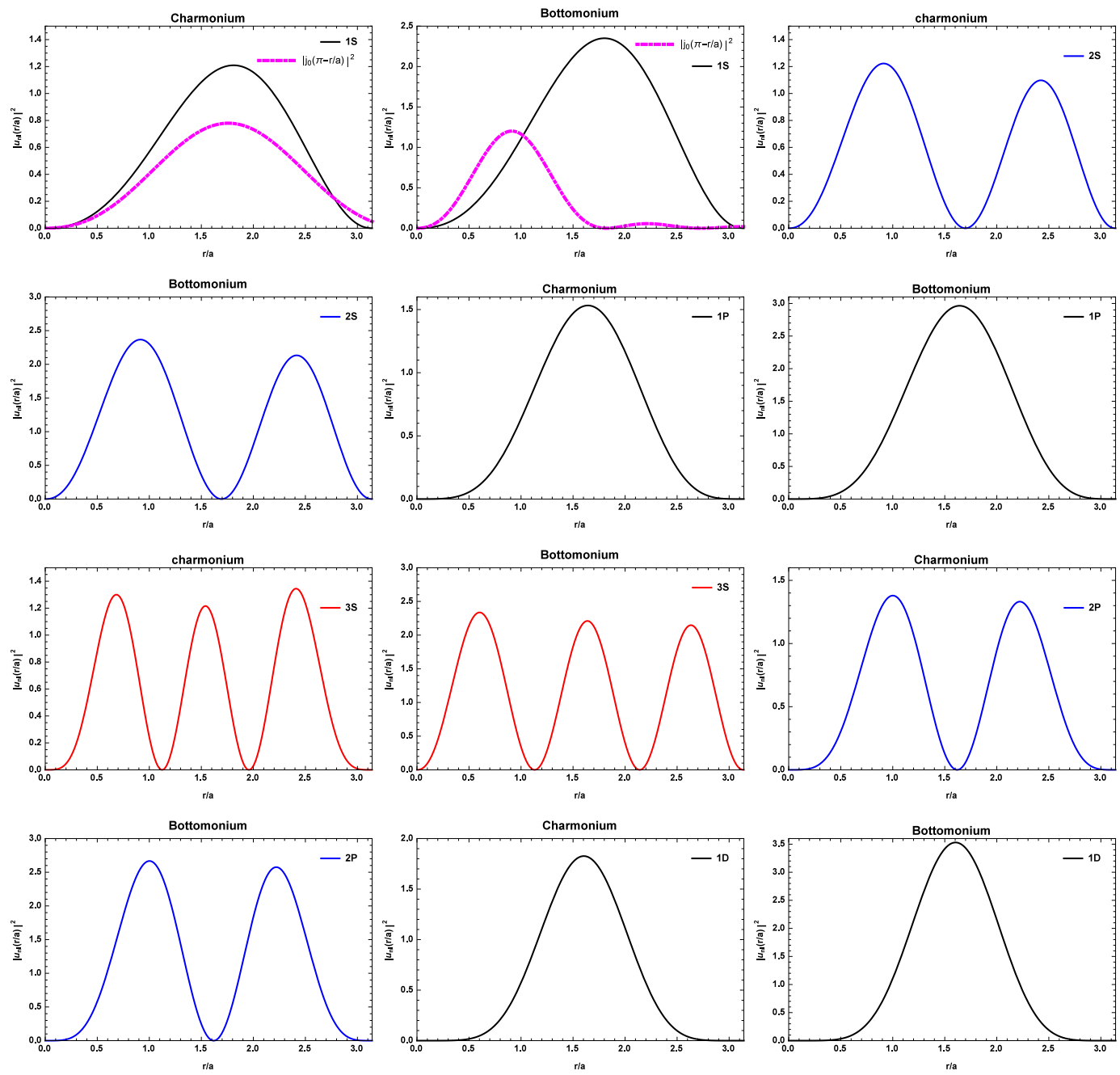

Figure 3: The normalized charmonium (left) and bottomonium (right) radial probabilities $\left|u_{n \ell}(r / a)\right|^{2}$ corresponding from top to bottom to $1 S, 2 S, 1 P, 3 S, 2 P$ and $1 D$ states, respectively. As a comparison, the spherical Bessel function $\left|j_{0}(\pi-r / a)\right|^{2}$, the ground state solution of the infinite spherical potential, has been plotted along with the $1 \mathrm{~S}$ states. 


\subsection{Root mean square radii}

Another quantity of physical interest is the root mean square radius (r.m.s.) $\left\langle r^{2}\right\rangle^{1 / 2}$ for a quark and anti-quark bound state. It it defined as

$$
\left\langle r^{2}\right\rangle^{1 / 2}=a\left[\int_{0}^{\pi}\left|u_{n l}\left(\frac{r}{a}\right)\right|^{2}\left(\frac{r}{a}\right)^{2} d\left(\frac{r}{a}\right)\right]^{1 / 2} .
$$

The calculated $\left\langle r^{2}\right\rangle^{1 / 2}$ values for several quarkonium states, using the potential parameters from the mass fit, are summarized in Table 3 . The results show little variation of this quantity over different states. We also noticed from Table 3 that the charm-to bottom root mean square radii are in a ratio of,

$$
\left\langle r^{2}\right\rangle_{c \bar{c}}^{1 / 2}:\left\langle r^{2}\right\rangle_{b \bar{b}}^{1 / 2} \approx 2: 1
$$

In order to get a better insight into the effect of $d$ on the r.m.s., we consider as a particular case the $1 S$ states and use in (58) the small angle approximation for the sine function given by, $\sin ^{\nu}(r / a) \approx(r / a)^{\nu}$. In addition, we extend the integration to the mathematically allowed infinity, with the aim of obtaining an expression in closed form. In so doing, we arrive at the following result,

$$
\begin{aligned}
\left\langle r^{2}\right\rangle^{1 / 2} & \approx \int_{0}^{\infty} d r N_{00}^{2} r^{2}\left(\frac{r}{a}\right)^{2-2 d} \exp \left(-\frac{2 r V_{0}}{2 a(d+1)}\right) \\
& =N_{00}^{2}\left(\frac{1}{a}\right)^{2-2 d} \Gamma(5-2 d)\left(\frac{V_{0}}{a d+a}\right)^{2 d-5} .
\end{aligned}
$$

Along same lines, the normalization constant $N_{00}$ defined by Eq. (55), now calculates as,

$$
N_{00} \approx\left[\left(\frac{1}{a}\right)^{2-2 d} \Gamma(3-2 d)\left(\frac{V_{0}}{a d+a}\right)^{2 d-3}\right]^{-1 / 2}
$$

Substituting this in Eq. (60), and then expanding around $d=0$ amounts to,

$$
\left\langle r^{2}\right\rangle=\frac{3 a^{2}}{\pi V_{0}^{2}}+\frac{5 a^{2} d}{2 \pi V_{0}^{2}}-\frac{3 a^{2} d^{2}}{\pi V_{0}^{2}} .
$$

The latter expression, and for the parameters in Tables 1, leads to $\left\langle r^{2}\right\rangle^{1 / 2}=0.947 \mathrm{fm}$ for $c \bar{c}$ and $0.494 \mathrm{fm}$ for $b \bar{b}$, respectively. Their ratio amounts to,

$$
\frac{\left\langle r^{2}\right\rangle_{c \bar{c}}^{1 / 2}}{\left\langle r^{2}\right\rangle_{b \bar{b}}^{1 / 2}}=1.92
$$

a value that is pretty close to the experimental one given in (59). Because of the smallness of the $d$ parameter, the equation (63) in combination with (62) clearly shows that the major part of the ratio in (59) is predominantly provided by the ratio of the corresponding $a$ parameters, which is $\left.a\right|_{c \bar{c}}:\left.a\right|_{b \bar{b}}=1.93$. We interpret this result as a stronger localization of the $b \bar{b}$ system relative to the $c \bar{c}$ system.

It is important to emphasize that mass spectra and r.m.s. radii have been calculated by one and the same parameter set $a, d, V_{0}, \mu$.

\section{Conclusions}

In this work, we obtained the energy eigenvalues and the corresponding eigenfunctions for the hard-wall trigonometric Rosen-Morse potential in (11) using the Nikiforov-Uvarov method. The expressions in closed forms for 
Table 3: The $\left\langle r^{2}\right\rangle^{1 / 2}$ (in fm) of $c \bar{c}$ and $b \bar{b}$ for various states. Other theoretical predictions are taken from [25]

\begin{tabular}{c|cccc}
\hline$n L$ & This work $c \bar{c}$ & From [25] $c \bar{c}$ & This work $b b$ & From [25] $b b$ \\
\hline 1S & 0.848 & - & 0.438 & 0.2 \\
1P & 0.890 & 0.7 & 0.457 & 0.4 \\
1D & 0.889 & 1.0 & 0.460 & 0.4 \\
2S & 1.001 & 0.9 & 0.512 & 0.5 \\
3S & 1.034 & 1.4 & 0.522 & 0.8 \\
3P & 0.959 & 1.8 & 0.506 & 1.0 \\
2D & 0.916 & 1.5 & 0.485 & 0.8 \\
1F & 0.888 & - & 0.461 & - \\
\hline
\end{tabular}
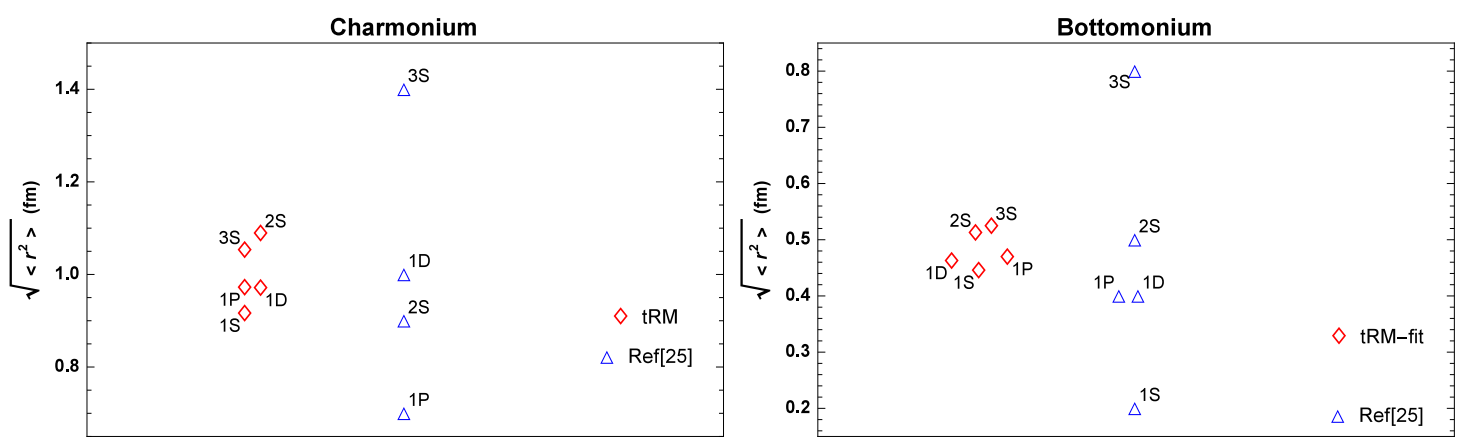

Figure 4: The root mean square radius for charmonium (left panel), and bottomonium (right panel). Our results are compared with other data taken from [25]. 
wave functions for different states have been explicitly given in the eqs. (49, 54). The corresponding reduced probability densities have been plotted in Fig. 3, and compared with the spherical Bessel function $j_{0}$, the ground state solutions that appear in the hard sphere method. As discussed after Eq. (17), the potential of interest allows for an interpretation as a conformal symmetry perturbing strong interaction, a topic of interest in the heavy flavor sector. The scheme has been applied to the $(c \bar{c})$ and $(b \bar{b})$ quarkonia and the results obtained on their mass spectra and root mean square radii have been compared to data, finding quite satisfactory agreements. Mass spectra and root mean square radii have been fairly well described by means of a Hamiltonian of moderately perturbed conformal symmetry, as signaled by the smallness of the symmetry violating parameter $d$, a fact that hints on the validity of a dynamically realized conformal symmetry in the heavy flavor sector. The dynamical realization of the symmetry under discussion allowed us to predict the quantum numbers appearing in the spectra and the splittings between the states in a level. The method predicted all correct signs of the splittings among the states in the levels, although strongly underestimated their magnitudes. We expect to find this situation improved by accounting for the relativistic kinematic level splittings as they would arise in employing the potential under discussion in a Klein-Gordon equation, a goal for a future research. We also were able to realistically describe the root mean square radii of the $c \bar{c}$ and $b \bar{b}$ systems, finding them in a ratio very close to $2: 1$, a fact which we interpreted as a stronger localization of the $b \bar{b}$ mesons relative to the $c \bar{c}$ mesons.

Our major conclusion is that conformal symmetry seems to remain present as a viable dynamical symmetry in all regimes of QCD. As a reminder, dynamical symmetry realization allows for degeneracy removal inside any one of the multiplets but forbids mixing among the multiplets [20]. Moreover, our conformal symmetry perturbing parameter $d$ in (17) features a realistic mass dependence in so far as it increased in the heavier bottomonium sector by a factor of about 1.6 relative to value characterizing the lighter charmonium sector, as it should be.

\section{References}

[1] Hsiang-nan Li, Guey-Lin Lin, and Wei-Min Zhang, Proceedings of the Fifth International Workshop Particle Physics Phenomenology, World Scientific Publishing Co. Pte. Ltd (2001).

[2] N. Brambilla et.al, Heavy Quarkonium Physics, CERN Yellow Report, CERN-2005-005, Geneva: CERN, 2005.- 487 p.

[3] N. Brambilla, Current Topics in Heavy Quarkonium Physics, arXiv:1106.1051v1 [hep-ph].

[4] A. Andronic et al., Heavy-flavour and quarkonium production in the LHC era: from protonproton to heavyion collisions, Eur. Phys. J. C 76, no. 3, 107 (2016).

[5] A. Deur, V. Burkert, J. P. Chen, and W. Korsch, Determination of the effective strong coupling constant $\alpha_{S, g_{1}}\left(Q^{2}\right)$ from CLAS spin structure function data, Phys. Lett. 665, 349 (2008).

[6] N. Rosen and Philip M. Morse, On the Vibrations of Polyatomic Molecules, Phys. Rev. 42, 210 (1932).

[7] M. Kirchbach and C. B. Compean, Modelling duality between bound and resonant meson spectra by means of free quantum motions on the de Sitter space-time $d S_{4}$, Eur. Phys. J A 52210 (2016). Addendum:M. Kirchbach, C. B. Compean, Eur. Phys. J. A 5365 (2017).

[8] V. V. Gritsev, Yu. A. Kurochkin, Model of excitations in quantum dots based on quantum mechanics in spaces of constant curvature, Phys. Rev. B, 64035308 (2001).

[9] Jean-Michel Caillol, Martin Trulsson, A new dipolar potential for numerical simulations of polar fluids on the 4D hypersphere, J. Chem. Phys. 141, 124111 (2014).

[10] M. Kirchbach and C.B. Compean, Protons electromagnetic form factors from a non-power confinement potential, Nucl. Phys. A (2018). 
[11] C.B. Compean and M. Kirchbach, The trigonometric RosenMorse potential in the supersymmetric quantum mechanics and its exact solutions, J. Phys. A: Math. Gen. 39547 (2006).

[12] Hakan Ciftci, Richard L. Hall, Nasser Saad, Exact and approximate solutions of Schrödingers equation for a class of trigonometric potentials, Cent. Eur. J. Phys. 11(1), 37-48 (2013).

[13] S. A. S. Ahmed and L. Buragohain, Generation of new classes of exactly solvable potential from the trigonometric Rosen-Morse potential, Indian J. Phys. 84 (6), 741-744 (2010).

[14] Chun-Sheng Jia, Tao Chen, Liang-Zhong Yi, Shu-Rong Lin, Equivalence of the deformed RosenMorse potential energy model and Tietz potential energy model, J Math Chem 51:21652172 (2013).

[15] A F Nikiforov and V B Uvarov, Special Functions of Mathematical Physics, (Birkhauser,Basel, 1988).

[16] Alvaro P. Raposo, Hans J. Weber, David E. AlvarezCastillo, Mariana Kirchbach, Romanovski polynomials in selected physics problems, CEJP 5(3) 253284 (2007).

[17] Richa Rani, S.B. Bhardwaj, and Fakir Chand, Mass Spectra of Heavy and Light Mesons Using Asymptotic Iteration Method, Commun. Theor. Phys. 70179 (2018).

[18] M. Tanabashi et al. (Particle Data Group), Phys. Rev. D 98, 030001 (2018).

[19] K.A. Olive et. al. (Particle Data Group), Chinese Physics C Vol. 38, No. 9090001 (2014).

[20] Robert Gilmore, "Group Theory”, in Mathematical Tools for Physicists, ed. Michael Grinfeld (Wiley-VCH, 2015), Chpt 5.11-5.12.4.

[21] Tapashi Das, D. K. Choudhury, K. K. Pathak, RMS and charge radii in a potential model, Indian J Phys 90: 1307 (2016).

[22] Stephen Godfrey, Kenneth Moats, and Eric S. Swanson, B and Bs meson spectroscopy, Phys. Rev. D 94, 054025 (2016).

[23] Estia J. Eichten, Chris Quigg, Mesons with Beauty and Charm: Spectroscopy, Phys.Rev.D49:5845-5856,1994

[24] Y. Maezawa and P. Petreczky, Quark masses and strong coupling constant in 2+1 flavor QCD, Phys. Rev. D 94, 034507 (2016).

[25] P. González, Long-distance behavior of the quark-antiquark static potential. Application to light-quark mesons and heavy quarkonia, Phys. Rev. D 80, 054010 (2009). 